\title{
EFECTOS DEL CAMBIO DE PRECIOS DE GARANTÍA A PROCAMPO EN PRECIOS AL PRODUCTOR, SIN INCLUIR EFECTO DE IMPORTACIONES
}

\section{EFFECTS OF THE CHANGE FROM SUPPORT PRICE TO PROCAMPO ON PRODUCER PRICES, EXCLUDING IMPORTS EFFECT}

\author{
Juan Hernández Ortiz ${ }^{1 *}$ y Miguel A. Martínez Damián²
}

\begin{abstract}
${ }^{1}$ Área de Agronomía, Universidad Autónoma Chapingo. Km. 38.5 Carr. México-Texcoco. 56230,Chapingo, Edo. de México. Tel. 01 595 9521572. ${ }^{2}$ Programa de Economía, Colegio de Postgraduados. Km. 36.5 Carr. México-Texcoco. 56230, Montecillo, Texcoco, Edo. de México. Tel. 01 595 9520200 ext. 1841.

*Autor para correspondencia (jhdzo@yahoo.com.mx)
\end{abstract}

\section{RESUMEN}

En este trabajo se analizó el efecto que ha tenido el cambio de política de apoyos a la agricultura, de precios de garantía a apoyos directos al productor (PROCAMPO) en el costo de una canasta conformada por maíz, frijol, arroz, trigo y sorgo, al eliminar el efecto depresor en precios de las importaciones, mediante cuatro índices de precios diferentes. Los resultados mostraron que al retirar la política de precios de garantía, y libre del efecto de las importaciones en los precios, el costo de dicha canasta bajó en 56.38 \% bajo pagos PROCAMPO.

Palabras clave: Precios de garantía, política de precios, subsidios directos al productor.

\section{SUMMARY}

This paper studied the cost effect induced by the change in agriculture policy, from price supports to direct payment to farmers on the bundle composed by corn, bean, rice, wheat and sorghum; this analysis was done by means of four index prices while the imports depressing price effect is removed. Once the support price policy ended, results showed that free from the imports effect, the chosen bundle cost decreased by $56.38 \%$ under PROCAMPO.

Index words: Support prices, price policies, direct producer's subsidies.

\section{INTRODUCCIÓN}

En 1994 con la entrada de México al contexto de la economía internacional al formalizar el Tratado de Libre Comercio con América del Norte (TLCAN), se terminó una forma de intervención gubernamental en la economía, particularmente en la agricultura a través del establecimiento de los precios de garantía (García, 1979) ${ }^{1}$, para dar paso al establecimiento de nuevas políticas agrícolas como PROCAMPO (Programa de Apoyos Directos al Campo), Alianza para el Campo y las políticas de apoyo a la comercialización de ASERCA (Apoyos y Servicios a la Comercialización Agropecuaria). Junto a estas acciones se implementaron reformas en la economía mexicana, buscando que las leyes del mercado se impongan en la economía y crear condiciones que permitan la competencia entre agentes de los diferentes sectores de la economía.

Un objetivo principal de la política agrícola aplicada durante la fase de precios de garantía fue incrementar la producción agrícola para garantizar la producción de alimentos y materias primas baratas, y apoyar así la industrialización. Las medidas durante este periodo consistieron principalmente en subsidios directos a la producción, comercialización y transformación, a través de precios subvencionados y del crédito agrícola, además de protección ante importaciones de mercancías agropecuarias externas. Sin embargo, estas políticas agrícolas contribuyeron al descenso de la competitividad agropecuaria y del desarrollo rural. Al comenzar la década de 1980 la política económica cambió y se iniciaron reformas regulatorias y estructurales con la finalidad primordial de transformar una economía cerrada y orientada al mercado interno, a otra abierta e integrada a la economía internacional. En materia agropecuaria las transformaciones iniciaron en 1986 con la entrada de

\footnotetext{
${ }^{1}$ Según García (1979), el 12 de Mayo de 1977 se creó el Comité Técnico de Precios de Garantía y Variables de Comercialización de Productos del Campo.
} 
México al Acuerdo General de Aranceles y Comercio (GATT), se continuó con un programa del agro centrado en la promoción de los mercados. Con la entrada en vigor del Tratado de Libre Comercio de América del Norte en 1994 y de los acuerdos de la Ronda Uruguay del GATT en 1995, las reformas estructurales agrícolas se intensificaron, las empresas públicas de transformación y comercialización de productos agrícolas fueron privatizadas o liquidadas, los subsidios a la producción fueron eliminados y sustituidos por pagos directos a los productores, y se liberó el comercio exterior de casi todos los bienes agrícolas.

Según Yúnez (1998), algunos efectos de las reformas y del TLCAN serían que el comercio agrícola aumentaría considerablemente entre México y los Estados Unidos, que la eliminación de los precios de garantía y el TLCAN provocaría una reducción en los precios de los cultivos básicos (cebada, maíz, oleaginosas, sorgo y trigo); además, la competencia entre productores de México, Canadá y EE. UU. aumentaría la productividad y reduciría la producción nacional, y los agricultores sustituirían su producción de cultivos básicos por cultivos de exportación (hortalizas y frutas).

En este tema, Bartra (1995), Schwentesius y Gómez (1993, 2004) y Schwentesius et al. (1998) se concentraron en los efectos negativos que este cambio de política tendría sobre los productores (disminución de precio interno, menor participación de la producción doméstica en el total vendido, reducción de la capacidad instalada y, en general, menor actividad económica); sin embargo, ignoraron los posibles efectos positivos en los consumidores mexicanos derivados de esta nueva política agrícola (menores precios, mayor variedad en presentaciones, disponibilidad de calidades no producidas internamente y mayor excedente del consumidor).

Rubio y Vélez (1994) advirtieron que muchos productores de granos básicos dejarían esta actividad para dedicarse al cultivo de frutas y hortalizas debido a la falta de competitividad con la producción de EE. UU. y Canadá. Calva (1996) reportó que en valores per capita la producción agrícola en 1994 resultó inferior en $17.7 \%$ respecto a 1981, y la producción de los ocho principales cultivos declinó en $26.7 \%$ en 1994. De Janvry et al. (1995) predijeron una reducción de $25 \%$ en la producción de granos con este cambio de políticas, lo que implicaba una pérdida de 800000 empleos en este sector.

Los estudios de Rubio y Vélez (1994), Calva (1996) y De Janvry et al. (1995) sobre los impactos del TLCAN abarcaron aspectos específicos, como son la producción, el cambio en la estructura productiva y modificaciones en el patrón de cultivos; investigadores concluyeron implícitamente que el cambio de política de precios de garantía a apoyos directos al productor, provocó la reducción en producción de granos y oleaginosas debido a la falta de competitividad de México con relación a EE. UU. y Canadá. Sin embargo, se ignora la posibilidad que esta nueva política pueda generar un abaratamiento del gasto en la canasta de dichos productos.

Andrécy et al. (2004) hicieron una comparación del gasto en una canasta conformada por arroz, trigo, sorgo, maíz y frijol, con datos de 1965 al 2000, bajo dos políticas de precios agrícolas, la primera referente a los precios de garantía que estuvieron vigentes hasta 1993 vs. la de PROCAMPO a partir de 1994. Sin embargo, ignoraron el efecto de las importaciones, ya que de 1994 a la fecha México abrió su economía con el consecuente incremento de las importaciones de estos cereales.

El presente estudio reconsideró la evaluación del cambio de política, incluyó el efecto de las importaciones antes ignorado y actualizó los datos para medir el efecto del cambio de política en el costo de la mencionada canasta.

En general, un mayor monto de importaciones hace descender los precios en el país que las realiza. De acuerdo con la ley del precio único, un efecto de la eliminación de las barreras arancelarias y no arancelarias entre los países miembros del TLCAN sería la igualación de los precios relativos de bienes comerciables en los tres países. Los efectos de la apertura comercial, por tanto, pueden resumirse en un cambio en los precios relativos nacionales, lo que produce cambios en diferentes cantidades: las producidas, las consumidas, la demanda de factores de producción y la distribución del ingreso. Conforme se acercan las fechas de desgravación los precios entre los países socios han tendido a converger $\mathrm{y}$, eventualmente, los precios relativos de los bienes comerciables en los tres países serán presumiblemente los mismos.

Por estas razones, y puesto que las importaciones han crecido a partir de 1994, es importante considerar el efecto de éstas en el precio interno de la canasta de productos básicos; los cultivos considerados son arroz, frijol, maíz, sorgo y trigo. El objetivo de esta investigación fue evaluar el impacto del cambio de política agrícola de precios de garantía al de pagos directos (PROCAMPO) en los precios reales de maíz, trigo, frijol, sorgo y arroz y, por tanto en el gasto, al descontar el posible efecto de las importaciones. Al respecto se postuló que el gasto total en 
la canasta maíz, frijol arroz, trigo y sorgo a nivel productor, es menor bajo la política de pagos directos PROCAMPO que el gasto bajo la política de precios de garantía, a pesar de eliminar el efecto de las importaciones.

\section{MATERIALES Y MÉTODOS}

Se colectaron series de precios corrientes, volúmenes de producción e importaciones en toneladas y rendimiento (en $\mathrm{t} \mathrm{ha}^{-1}$ ) de: arroz, frijol, maíz, trigo y sorgo, índice de precios al consumidor base 1994, y población total. Tales series contienen datos de 1965 a 2003. Las fuentes de consulta fueron: el Sistema Agropecuario de Consulta SIACON (SAGARPA 2006), Organización de las Naciones Unidas para la Alimentación y Agricultura (FAO 2006), estadísticas históricas de México (ITAM) y Banco de México.

Dado que existe la posibilidad de que los precios de los productos, y por ende el del gasto, estén influenciados por el efecto depresor del precio interno de las importaciones, se eliminó este efecto. Para aislar lo se buscó un modelo que explicara los precios al productor de los productos en función de importaciones, cantidad producida y el rezago de los precios al productor. Estos modelos fueron una combinación de series de tiempo de los precios con otras variables como las importaciones y la cantidad interna producida, con el propósito de descontar el efecto de las importaciones en los precios, sin abordar un modelo estructural de demanda.

Esta forma de proceder obedeció a la imposibilidad de responder a un modelo específico para cada producto, en el que se consideren precios de bienes relacionados, estructura de oferta y saldo de comercio exterior. Por tanto, se eligió un modelo de precios que incorpora el efecto de importaciones y aproxima los aspectos de oferta y demanda no considerados explícitamente a través de un polinomio de bajo orden en rezagos de precios, cantidades producidas e importaciones. Greene (2000) denominó a estos modelos como de rezago distribuido.

Los modelos que se estimaron mediante el procedimiento MODEL de SAS fueron:

$P R_{i t}=a_{i}+\beta_{1 i} P_{i t-1}+\beta_{2 i} Q_{i t-1}+\beta_{3 i} I_{i t-1}+\varepsilon_{i t}$

Donde $\mathrm{i}=$ Arroz, trigo y sorgo.

$P_{i t}=a_{i}+\beta_{1 i} P R_{i t-1}+\beta_{2 i}\left(Q_{i}+I_{i}\right)_{t-1}+\varepsilon_{i t}$

Donde $\mathrm{i}=$ Maíz y frijol.

Note que la Ec. 2 es una forma restringida de la Ec. 1.
Para las Ecuaciones 1 y $2, \mathrm{PR}=$ precio real; $\mathrm{Q}=$ cantidad interna producida; $\mathrm{I}=$ importaciones; $\mathrm{t}=$ años; $\varepsilon_{\mathrm{t}}=$ término de error.

Con el propósito de evaluar el impacto de cambio de política libre del efecto importaciones, el precio predicho se obtuvo bajo la restricción de que las importaciones eran cero. Con base en las Ecuaciones 1 y 2, resultó la siguiente ecuación:

$P R_{i}=a_{i}+\beta_{1 i} P R_{t-1}+\beta_{2 i} Q_{i t-1}$

Se supuso que el coeficiente de las importaciones es de signo negativo, por lo que se espera que los precios predichos sean mayores cuando $\mathrm{I}=0$.

Con las series de precios reales predichos, sin los efectos de importaciones, se procedió a la comparación del costo de la canasta estudiada para cada política. Para ello se hizo uso de la metodología de índices de precios (Martínez, 1999). Los índices empleados son (Varian, 1998): Laspeyres, Paasche, Fisher y Tornqvist, con modificaciones para eliminar inflación y crecimiento económico con la metodología empleada por Andrécy et al. (2004).

En este estudio se establecieron los siguientes supuestos: 1) El análisis es a nivel productor; 2) No se consideran márgenes de comercialización; 3) No hay cambios en gustos y preferencias debido al cambio de política; 4) Ausencia de cambios tecnológicos, o de haberlos afectarán la canasta estudiada por igual; 5) La apertura comercial y las importaciones propician un descenso en los precios, por lo que se diseñó un mecanismo para aislar dicho efecto y 6) El cambio de política sólo afecta a través de precios. Se espera así medir el efecto del cambio de políticas agrícolas en el costo de la canasta considerada.

El efecto del cambio de política se evaluó con la metodología de un índice de precios (Varian, 1998) mediante el cual se puede comparar el costo de una canasta de bienes en dos periodos diferentes correspondientes a dos políticas agrícolas. Por ejemplo, el índice de precios Paasche, IPP $=\frac{\mathrm{P}^{\prime}{ }_{t} \mathrm{Q}_{\mathrm{t}}}{\mathrm{P}^{\prime}{ }_{0} \mathrm{Q}_{\mathrm{t}}}$, mide el cambio en el costo de una canasta comprada a distintos precios en el tiempo; como $\mathrm{Q}$ tiene un subíndice tiempo la canasta cambia. A su vez, el índice de Laspeyres, IPL = $\frac{\mathrm{P}_{t}^{\prime} \mathrm{Q}_{0}}{\mathrm{P}_{0}^{\prime} \mathrm{Q}_{0}}$, mide el efecto del gasto a través de lo que cueste la misma canasta en diferentes periodos; en este caso $\mathrm{Q}$ no cambia. En ambos índices la canasta de referencia es la 
misma, por lo que el efecto se da a través de los precios. El índice de precios de Fisher, IPF $=\sqrt{\mathrm{IPL} \times \mathrm{IPP}}$, incorpora el efecto sustitución, y el de Tornqvist aproxima a un índice verdadero. Al extender los índices Paasche y Laspeyres, también se calcula el índice del gasto $\mathrm{IGC}=$ $\frac{\mathrm{P}^{\prime} \mathrm{Q}_{\mathrm{t}}}{\mathrm{P}^{\prime}{ }_{\mathrm{o}} \mathrm{Q}_{0}}$. En cada caso, para eliminar el efecto del crecimiento económico aproximado por población se utiliza la siguiente expresión: $\mathrm{ICP}=\frac{\mathrm{POB}_{\mathrm{t}}}{\mathrm{POB}_{0}}$. En estas expresiones $\mathrm{P}_{\mathrm{t}}, \mathrm{P}_{0}$ son vectores de precios a los tiempos t y 0 respectivamente; por su parte $\mathrm{Q}_{t}, \mathrm{Q}_{0}$ son vectores de cantidad a los tiempos $\mathrm{t}$ y $0 ; \mathrm{y} \quad \mathrm{POB}_{\mathrm{t}}, \mathrm{POB}_{0}$ son las poblaciones a los tiempos t y 0 .

\section{RESULTADOS Y DISCUSIÓN}

Los modelos de importación arrojaron coeficientes pequeños para el efecto de importaciones en los precios (Cuadro 1). El efecto importaciones resultó negativo para todos los productos, lo que implica que a mayores importaciones menor será el precio real del producto examinado. Los coeficientes estimados correspondientes a importaciones y cantidades producidas también fueron muy pequeños; sin embargo, las flexibilidades precio $^{2}$ de las importaciones calculadas con base en los estimadores del Cuadro 1 , señalan que si las importaciones crecen en $1 \%$, porcentualmente el precio real interno disminuirá para maíz en 0.12 , frijol en 0.104 , arroz en 0.049 , trigo en 0.17 y sorgo en 0.14 . Estos valores indican que los precios de los cinco productos son poco sensibles a las importaciones de los mismos.

\section{Índices de precios y de gasto en términos reales}

En la Figura 1 se presentan los cambios en los índices de precios y del gasto calculados con precios reales de 1966 al 2003. En general, los índices de precio decrecieron en el transcurso de los años, sobre todo a partir de 1994, fecha en que ocurre la apertura comercial y se echan a andar las reformas en política agrícola, entre ellas PROCAMPO. A manera de ejemplo, el índice de precios Laspeyres en términos reales indica que de 1966 a 1993 el índice disminuyó en 49.5 \% y que de 1993 a 2003 los precios se redujeron en $66.7 \%$, lo que significa una tasa media anual de decrecimiento de 1.767 y $6.67 \%$

\footnotetext{
${ }^{2}$ La flexibilidad precio $\left(F=\frac{\partial \mathrm{P}}{\partial \mathrm{I}} \frac{\mathrm{I}}{\mathrm{Q}}\right)$ mide el cambio porcentual en el

precio de un bien ante un cambio de $1 \%$ en la cantidad demandada; análogamente, la flexibilidad precio de las importaciones mide el mismo cambio pero ante el incremento de $1 \%$ en la cantidad importada.
}

respectivamente. Es decir, la nueva política agrícola aceleró el abaratamiento de los productos estudiados.

En el gasto real durante la etapa de precios de garantía hubo un crecimiento de 1966 a 1993 (Figura 1), mientras que el índice decreció de 1994 a 2003, época de PROCAMPO, lo que muestra tendencias contrarias en las dos políticas agrícolas, según este indicador.

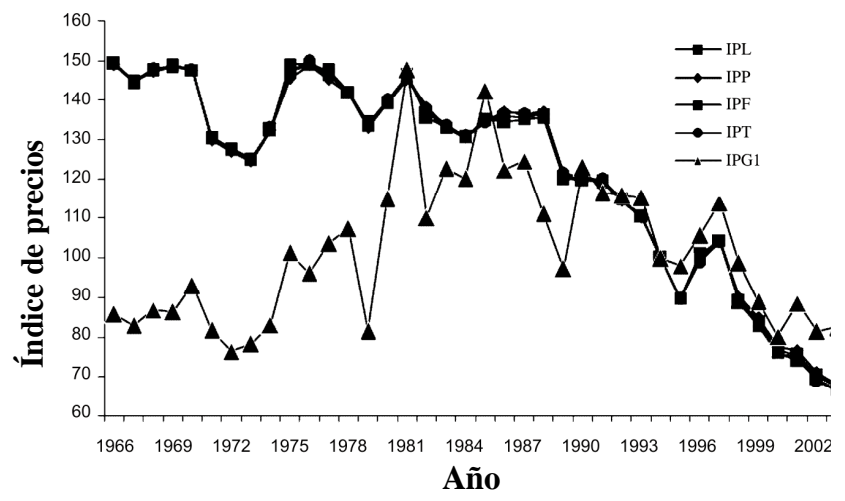

Figura1. Índices de precios $y$ gasto en el periodo 1966-2003, calculados con precios reales base 1994.

IPL = Índice de precios Laspeyres; IPP = Índice de precios Paasche; IPF = Índice de precios Fisher; IPT = Índice de precios Tornqvist; IPG1 = Índice del gasto Real.

Con los índices de precios calculados con precios reales para los años 1966 a 2003 se hizo una prueba de medias para probar la hipótesis $\mu_{\mathrm{pg}}=\mu_{\mathrm{pro}} S . \mu_{\mathrm{pg}}>\mu_{\mathrm{PRO}}$, donde $\mu_{\mathrm{pg}}$ es la media de precios bajo política de precios de garantía

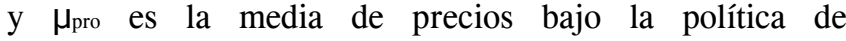
PROCAMPO. En el Cuadro 2 se muestra que cuando se tomaron 28 datos para precios de garantía y 10 para PROCAMPO, las medias de precios de garantía resultaron ser mayores en todos los casos $(\mathrm{P} \leq 0.05)$. El contraste de medias se acompañó con una prueba de homogeneidad de varianzas, misma que no se rechazó con excepción del índice de gasto real. Del Cuadro 2 se concluye que el costo de la canasta de productos estudiados resultó ser más alto en la época de precios de garantía que en la época de la política de pagos directos vía PROCAMPO.

Cuando se consideraron tamaños de muestras iguales, 10 años de precios de garantía y 10 de PROCAMPO, se concluyó que la diferencia es positiva entre ambos periodos, aun al eliminar el efecto importaciones. Es decir, los precios sufrieron un descenso durante el periodo de pagos directos comparados con el periodo de precios de garantía, y dicho descenso no se debe a importaciones. La prueba de homogeneidad de varianzas no fue rechazada en cada caso alguno. 
Cuadro 1. Parámetros estimados para eliminar el efecto de importaciones en el precio doméstico.

\begin{tabular}{lccccc}
\hline Modelo & Ordenada al origen & Coeficiente de $Q_{i}-1$ & Coeficiente de $I_{i}-1$ & $R^{2}$ & Durbin Watson \\
\hline Arroz & $1081.345 \pm 442.4$ & $-0.00129 \pm 0.000478$ & $-0.00031 \pm 0.000880$ & 0.8614 & 1.6388 \\
Trigo & $2753.428 \pm 188.4$ & $-0.00045 \pm 0.000053$ & $-0.00014 \pm 0.000057$ & 0.8514 & 1.6772 \\
Sorgo & $1204.327 \pm 167.2$ & $-0.00008 \pm 0.000043$ & $-0.00005 \pm 0.000045$ & 0.8052 & 1.6780 \\
Maíz & $1418.022 \pm 144.5$ & $-0.00005 \pm 9.126 \mathrm{E}-6$ & $-0.00005^{\dagger} \pm 9.126 \mathrm{E}-6$ & 0.9139 & 1.6495 \\
Frijol & $6195.856 \pm 1198.3$ & $-0.00281 \pm 0.00104$ & $-0.00281^{\dagger} \pm 0.00104$ & 0.4511 & 1.6458
\end{tabular}

Fuente: Elaboración propia (los números entre paréntesis son los error estándares de los estimadores). $\dagger$ Valores correspondientes a $\beta_{2 \mathrm{i}}$.

Cuadro 2. Comparación de medias con muestras de tamaño igual y diferente.

\begin{tabular}{|c|c|c|c|c|c|c|c|}
\hline Variable & Política & $\mathrm{N}$ & Media & Desv. est. & GL & Valor de t & $\operatorname{Pr}>|t|$ \\
\hline IPL & PG & 28 & 135.27 & 11.36 & 36 & 11.35 & $<.0001$ \\
\hline IPL & PRO & 10 & 85.27 & 13.61 & & & \\
\hline IPL & G & 10 & 125.57 & 9.65 & 18 & 7.64 & $<.0001$ \\
\hline IPP & PG & 28 & 135.14 & 11.07 & 36 & 11.57 & $<.0001$ \\
\hline IPP & PRO & 10 & 86.26 & 12.59 & & & \\
\hline IPP & PG & 10 & 126.20 & 10.17 & 18 & 7.81 & $<.0001$ \\
\hline IPF & PG & 28 & 135.20 & 11.20 & 36 & 11.47 & $<.0001$ \\
\hline IPF & PRO & 10 & 85.76 & 13.09 & & & \\
\hline IPF & PG & 10 & 125.88 & 9.89 & 18 & 7.73 & $<.0001$ \\
\hline IPT & PG & 28 & 135.55 & 11.22 & 36 & 11.59 & $<.0001$ \\
\hline IPT & PRO & 10 & 85.15 & 13.40 & & & \\
\hline IPT & PG & 10 & 126.22 & 9.90 & 18 & 7.8 & $<.0001$ \\
\hline IPGR* & PG & 28 & 104.67 & 19.47 & 27.9 & 2.11 & 0.044 \\
\hline IPGR* & PRO & 10 & 93.86 & 11.27 & & & \\
\hline IPGR & PG & 10 & 118.90 & 11.40 & 18 & 4.94 & 0.0001 \\
\hline
\end{tabular}

Fuente: Elaboración propia. $\mathrm{PG}=$ Precios de garantía; PRO = PROCAMPO; $\mathrm{n}=28$ en los años de PG; $\mathrm{n}=10$, los últimos 10 años. En la comparación de medias se rechazó la prueba de homogeneidad de varianzas ${ }^{1}$, lo que se tomó en cuenta al momento del contraste. IPL, IPP, IPF, IPT e IPGR son los índices de precios señalados en Figura 1.

Cuadro 3. Comparación de medias con muestras de tamaño igual y diferente en índices y gasto real per capita.

\begin{tabular}{|c|c|c|c|c|c|c|c|}
\hline Variable & Política & $\mathrm{N}$ & Media & Desv. est. & GL & Valor de t & $\operatorname{Pr}>|t|$ \\
\hline IPLP & PG & 28 & 202.22 & 5650 & & & \\
\hline IPLP & PRO & 10 & 79.56 & 16.38 & 35.3 & 10.34 & $<.0001$ \\
\hline IPLP & PG & 10 & 144.46 & 21.09 & 18 & 7.69 & $<.0001$ \\
\hline IPPP & PG & 28 & 201.90 & 55.85 & & & \\
\hline IPPP & PRO & 10 & 80.43 & 15.46 & 35 & 10.44 & $<.0001$ \\
\hline IPPP & PG & 10 & 145.20 & 21.53 & 18 & 7.73 & $<.0001$ \\
\hline IPFP & PG & 28 & 202.06 & 56.17 & & & \\
\hline IPFP & PRO & 10 & 79.99 & 15.92 & 35.2 & 10.39 & $<.0001$ \\
\hline IPFP & PG & 10 & 144.83 & 21.30 & 18 & 7.71 & $<.0001$ \\
\hline IPGP $^{\dagger}$ & PG & 28 & 150.21 & 22.31 & & & \\
\hline IPGP $^{\dagger}$ & PRO & 10 & 87.34 & 14.16 & 36 & 8.29 & $<.0001$ \\
\hline IPGP & PG & 10 & 136.59 & 20.57 & 18 & 6.24 & $<.0001$ \\
\hline
\end{tabular}

Fuente: Elaboración propia. $\dagger$ No rechaza la hipótesis de varianzas homogéneas. PG = Precios de garantía; PRO = PROCAMPO. IPL, IPPP, IPFP, IPT e IPGP son los índices de precios indicadores en Figura 2.

\footnotetext{
${ }^{1}$ El estimador de la varianza es una media ponderada de las varianzas de las dos muestras, con más peso a la muestra más grande definido como $\mathrm{s}^{2}=\left(\left(\mathrm{n}_{1}-\right.\right.$ $\left.1) \mathrm{s}^{2} 1+\left(\mathrm{n}_{2}-1\right) \mathrm{s}^{2}\right) /\left(\mathrm{n}_{1}+\mathrm{n}_{2}-2\right)$, donde $\mathrm{s}^{2}{ }_{1} \mathrm{y} \mathrm{s}^{2} 2$ son las varianzas de las muestras, $\mathrm{n}_{1} \mathrm{y} \mathrm{n}_{2}$ el tamaño de muestras. El error estándar de la media de la diferencia es la varianza así estimada ajustada por el tamaño de muestra. Satterthwaite es una alternativa de otro estimador de la varianza en la prueba de $t$ y es usada cuando el supuesto de homogeneidad de varianzas es insostenible; éste da una estadística t que se aproxima asintóticamente a la distribución t conforme la muestra es más grande, lo que permite realizar la prueba t aun cuando las varianzas de las poblaciones difieran.
} 


\section{Índices y gasto en términos reales per capita.}

El mismo resultado se obtiene si se elimina el efecto de la inflación y se normaliza por el crecimiento poblacional, para eliminar el efecto del crecimiento económico, por tanto, el análisis se repite con variables reales per capita. En este caso, la Figura 2 muestra que la disminución de precios promedio anual fue similar bajo ambas políticas. Sin embargo, cuando se trata del gasto real per capita la disminución media anual fue mayor durante el periodo PROCAMPO; en particular en el periodo 1993 a 2003 el gasto real per capita cayó en $54.1 \%$ mientras que en el periodo 1983 a 1993 sólo lo hizo en $43.5 \%$, lo cual significa que el gasto que realiza un productor en esta etapa está disminuyendo más aceleradamente que en el periodo de precios de garantía.

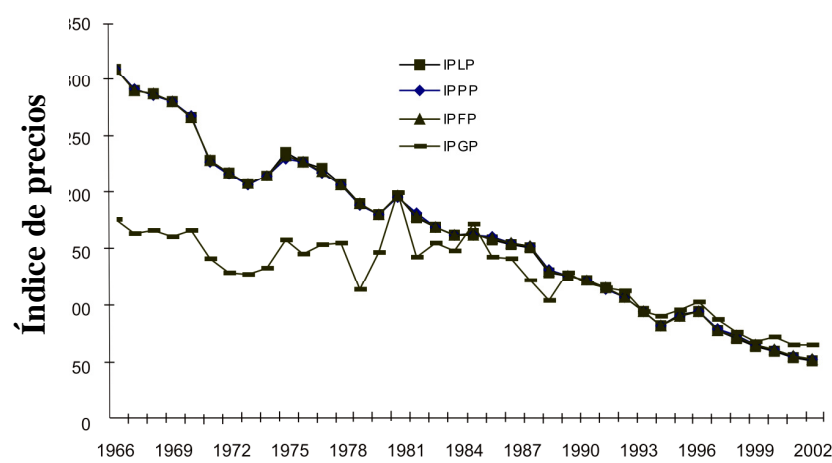

Año

Figura 2. Índices de precios y gasto per cápita en el periodo 19662003, con precios reales base 1994.

Fuente: Elaboración propia: IPLP = Índice de precios Laspeyres; IPPP = Índice de precios Paasche; IPFP = Índice de precios Fisher; IPGP = Índice del gasto Real. Todos son per capita.

Al comparar las medias (Cuadro 3) con muestras de 28 datos (precios de garantía) y 10 datos para PROCAMPO así como de 10 datos para cada periodo, se encontró que: con 28 y 10 datos para precios de garantía y PROCAMPO respectivamente, en la variable IPGP no se rechazó la prueba de varianzas homogéneas, mientras para todas las otras variables sí se rechazó la hipótesis de varianzas homogéneas. En cambio, cuando las muestras estaban balanceadas no se rechazó la hipótesis de varianza homogénea en ningún caso.

En ambos tamaños de muestras con significancia estadística, las medias de los índices de precios y del gasto per capita fueron mayores en el periodo de precios de garantía, lo cual confirma que una política de control de precios encarece el gasto del consumidor, comparado con la política de pagos directos implementada en los últimos diez años de los datos analizados.

\section{CONCLUSIONES}

$\mathrm{Al}$ analizar el comportamiento de los precios anteriores y posteriores al inicio de la política de pagos directos (PROCAMPO), y una vez eliminado el efecto importaciones, se concluyó que el costo de la canasta de productos básicos (maíz, frijol, arroz, trigo y sorgo) tendió a disminuir en el tiempo. Esta tendencia se acentuó a partir de 1994, fecha en que inició el programa PROCAMPO y el TLCAN. El costo de dicha canasta fue menor en el periodo PROCAMPO que en el de precios de garantía. La política de pagos directos resultó en un beneficio para los compradores de dichos productos a nivel productor. Las importaciones han tenido un efecto negativo en los precios de los productos de la canasta estudiada.

\section{BIBLIOGRAFÍA}

Andrécy E R H, M A Martínez D (2004) Apoyos directos contra precios de garantía: un enfoque de bienestar (gasto). Claridades Agropecuarias 134, octubre 2004. Revista mensual producida y editada por Apoyos y Servicios a la Comercialización Agropecuaria, de la Secretaría de Agricultura, Ganadería, Desarrollo Rural, Pesca y Alimentación, México.

Bartra A (1995) Un PROCAMPO devaluado. De los precios de garantía a los precios de castigo. Jornada del Campo 34:10-11. http://www.banxico.org.mx/tipo/estadisticas/index.html

Calva J L (1996) La reforma económica de México y sus impactos en el sector agropecuario. In: El Campo Mexicano, una Modernización a Marchas Forzadas. P. Bovin (comp). Centro Francés de Estudios Mexicanos y Centroamericanos. México.

De Janvry A, E Sadoulet, B Davis (1995) NAFTA's impact on Mexico household-level effects. Amer. J. Agric. Econ. 77:1283-1291. Disponible en: http://www.fao.org/corp/statistics/es/ 2006

García M R (1979) Análisis Sobre Precios de Garantía. Centro de Economía Agrícola, Chapingo México. 32 p.

Greene W H 2000 Econometric Analysis. 4 th ed. Prentice Hall. USA. $1004 \mathrm{p}$.

ITAM (2006) Estadísticas Históricas de México. Agricultura, ganadería, pesca y silvicultura. Disponible en: http://biblioteca.itam.mx

INEGI (1965-1997) Estadísticas Históricas. México.

Martínez D M A (1999) Introducción al uso del índice de precios Tornqvist. Agrociencia 33:179-182.

Rubio G M, F Vélez (1994) El impacto del TLC en el campo mexicano. In: Lo Negociado del TLC, el Impacto Sectorial del TLC en la Economía Mexicana. G Kessel (comp). Mac Graw Hill. México. pp.

SAGARPA (2006) Sistema Agropecuario de Consulta (SIACON). 19802005. México.

Schwentesius R, M A Gómez C (1993) Política agrícola hacia el siglo XXI. PROCAMPO y Alianza para el Campo en el contexto internacional. CIESTAM, UACh, Chapingo, México.

Schwentesius R, M A Gómez C (2004) TLCAN y Agricultura Mexicana: Lecciones a 10 Años. CIESTAM, UACh, Chapingo, México.

Schwentesius R, M A Gómez C, G Williams (1998), Coordinadores: TLC y Agricultura; ¿Funciona el Experimento? CIESTAM, UACh, México.

SNIIM (2006) Servicio Nacional de Integración e Información de Mercados. Disponible en: www.economía-sniim.gob.mx/

Varian H. (1998) Análisis Macroeconómico. 3ra ed. Editorial Antoni Bosch. Barcelona, España pp:125-26 y 160-61. 
Yúnez A (1998) El TLC, las reformas del cambio estructural y la agricultura mexicana. In: TLC y Agricultura; ¿Funciona el Experimento? Schwentesius, M A Gómez C, G Williams (eds). CIESTAM, UACh, México. 